\title{
Metabolomic Investigation on Fermentation Products of Achyranthes japonica Nakai by Lactobacillus plantarum
}

\author{
Chang-Wan Lee ${ }^{1,2}$ and Do Yup Lee ${ }^{3 *}$ \\ ${ }^{1}$ Department of Bio and Fermentation Convergence Technology, BK21 PLUS Program, Kookmin University, Seoul 02707, Republic of Korea \\ ${ }^{2}$ Bio RED Center, SK Bioland Co., Ltd., Ansan 15407, Republic of Korea \\ ${ }^{3}$ Department of Agricultural Biotechnology, Center for Food and Bioconvergence, Research Institute for Agricultural and Life Sciences, Seoul \\ National University, Seoul 08826, Republic of Korea
}

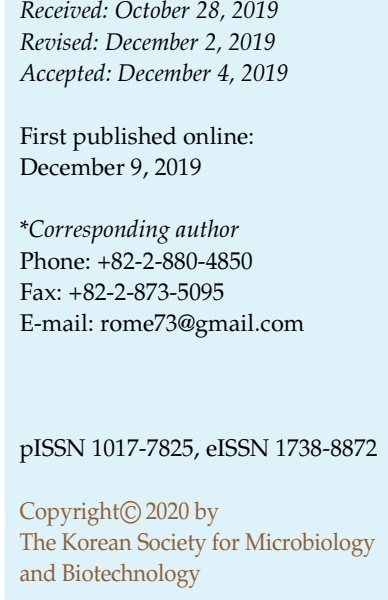

Fermentation has recently re-emerged as an approach for improved functionality of food products in addition to the traditional roles such as shelf life, taste, and texture. Here, we report dynamic changes in the metabolite profiles of Achyranthes japonica Nakai by Lactobacillus plantarum fermentation, primarily, the significant increases in representative functional ingredients, 20-hydroxyecdysone and 25S-inokosterone. Additionally, untargeted metabolite profiling showed $58 \%$ of metabolites underwent significant alteration. The most dynamic change was observed in cellobiose, which showed a 56-fold increase. Others were sugar alcohols and amino acids, while lyxitol and erythritol that were among the most dynamically down-regulated.

Keywords: Achyranthes japonica Nakai, Lactobacillus plantarum fermentation, LC-orbitrap MS, GC-TOF MS, metabolic profiling
Achyranthis Radix is an herbal medicine derived from Achyranthes bidentata Blume and Achyranthes japonica Nakai (AJN), which has been recognized for improved sinew and bone health. Recent related scientific evidence has also reported hypoglycemic, anti-inflammatory, and antihyperlipidemic activities [1].

Our current study aims at improving the nutraceutical and nutritional quality of whole extracts of AJN by Lactobacillus plantarum fermentation. Lactic acid bacteria are the best-known microorganisms associated with food fermentation, and their capability to metabolize a range of plant-derived compounds has been investigated [2, 3]. A recent study applied microbial fermentation for cherry juice by Lactobacilli, which resulted in potential benefits including decreased sugar content, improved flavor, and increased bioactivities.

To comprehensively profile the fermentation-driven metabolic features of AJN, integrative mass-spectrometric analysis was conducted based on targeted and untargeted profiling approaches. We analyzed 20-hydroxyecdysone and 25S-inokosterone, two representative bioactive compounds, using liquid-chromatography orbitrap mass-spectrometry (LC-orbitrap MS). In addition, a range of primary metabolic profiles were acquired using gas-chromatography time-offlight mass spectrometry (GC-TOF MS). The results demonstrated the substantial modulation in the metabolite contents of AJN following the fermentation process including the major bioactive markers.

We first examined if the fermentation affected the level of 20-hydroxyecdysone, major indicator and functional compound of AJN $[1,4,5]$. Reverse phase liquid chromatography coupled to mass-spectrometric analysis was conducted with positive ionization mode.

Two extracted ion chromatograms were detected at $\mathrm{m} / \mathrm{z}$ of 481.3165 (retention time: $1.41 \mathrm{~min}$ and $2.67 \mathrm{~min}$ ) with mass accuracy of $5 \mathrm{ppm}$. The $\mathrm{m} / \mathrm{z}$ value corresponded to $[\mathrm{M}+\mathrm{H}]^{+}$ion of 20-hydroxyecdysone and 25S-inokosterone, and the identities were differentiated by tandem mass spectra and retention time [1,6] (Fig. 1A). Quantitative comparison (peak area) of the compounds showed the significant increase following the post-fermentation process (fold change $=2.6$ and 2.3, respectively for 20-hydroxyecdysone 
A

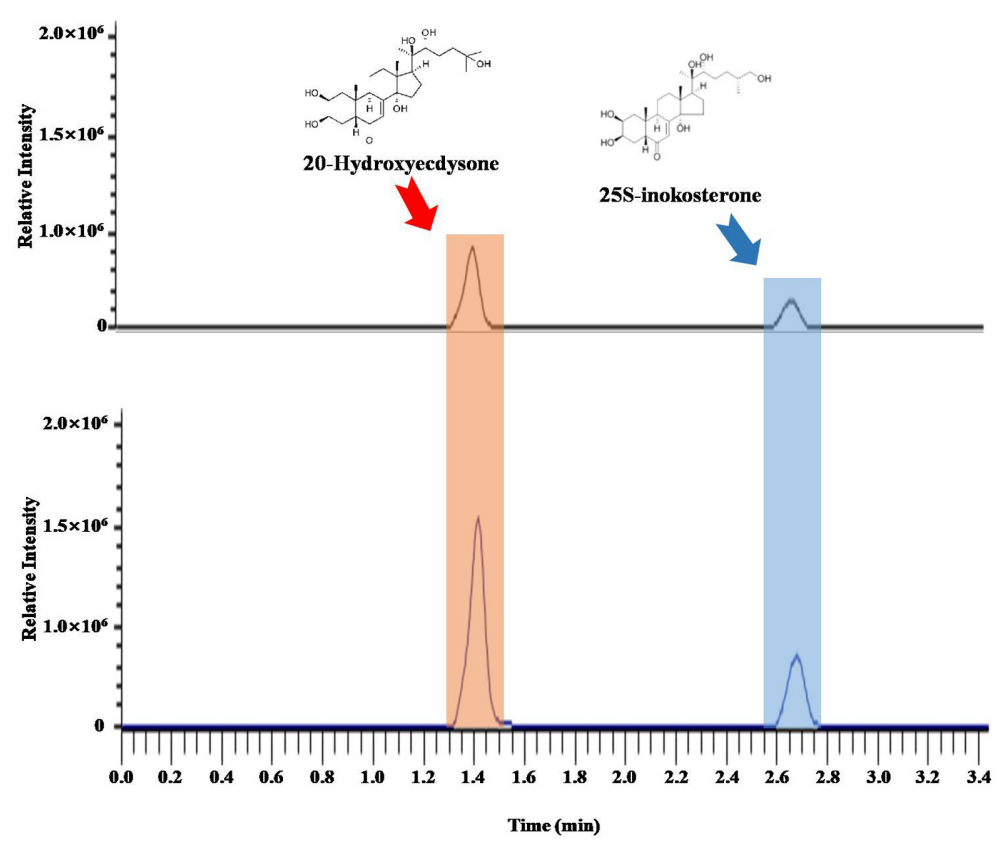

B
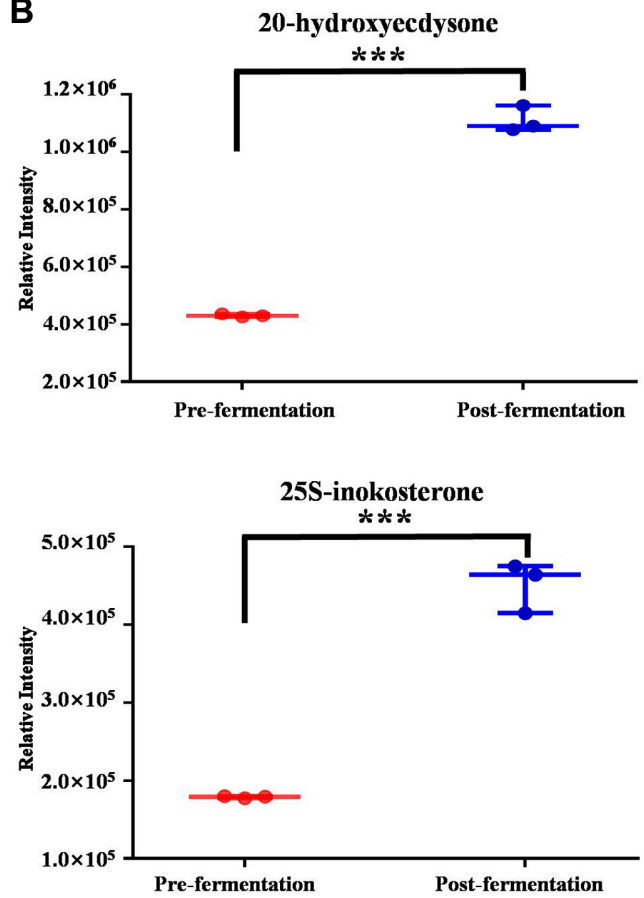

Fig. 1. The significant increases of primary indicator and representative functional compounds, 20-hydroxyecdysone and 25Sinokosterone.

(A) Base peak chromatograms and (B) semi-quantitative comparison of the compounds between pre- and post-fermentation. Statistical analysis was done based on Student's $t$-test. ${ }^{* * *} p<0.001$. Y-axis is relative intensity.

and 25S-inokosterone, $p<0.001$ ) (Fig. 1B).

GC-TOF MS analysis was performed on extract derivatives to identify additional changes in the AJN metabolome by the Lactobacillus plantarum fermentation. The untargeted metabolic profiling resulted in 80 compounds that were uniquely identified and semi-quantified based on Binbase algorithm [7]. The metabolites were carbohydrates (34\%), amino acids $(28 \%)$, organic acids $(10 \%)$, fatty acids $(8 \%)$, and others (20\%).

First, principal component analysis (PCA) revealed clear distinction in the metabolite profiles between pre- and post-fermentation of the AJN. T1 separated the two groups $(64.6 \%)$ while T2 mostly explained the variance among biological replicates (different batches) within each group $(80.4 \%)$ (Fig. 2A). Likewise, the subsequent model using PLS-DA presented the clear discrimination with best explained variance $\left(R^{2} Y=1\right)$ and predictability $\left(Q^{2}=0.993\right)$ (Fig. 2B). The VIP analysis showed that TCA cycle intermediates, malic acid and fumaric acid most contributed to the discriminant model. Others were organic acids (glucuronic acid and glyceric acid), nitrogenous compounds (valine, 3hydroxypyridine, xanthine, and thymine), cellobiose, and lyxitol.
Next, the compositional characteristics after the fermentation process were further investigated using univariate statistics (Student's $t$-test). A total of 46 metabolites were significantly different in the fermented extract in which 36 and 10 compounds were up- and down-regulated (Table 1). The highest fold-increase was found in cellobiose (55.7 fold-change, $p<0.001)$ whereas the most dynamic downregulation was detected in lyxitol ( 0.1 fold-change, $p<$ 0.001). Pathway over-representation analysis proposed that amino acid metabolisms were most significantly altered by the fermentation, which included Ala-Asp-Glu metabolism, Val-Leu-Ile metabolism, Arg-Pro metabolism, and Tyr metabolism (Fig. 2C). Other metabolisms were pantothenateCoA biosynthesis, carbon fixation, and butanoate metabolism. In addition, we explored the putative biochemical mechanism on the increased levels of 20-hydroxyecdysone. We retrospectively investigated potential precursors or intermediates that may be converted to 20-hydroxyecdysone by the Lactobacillus plantarum fermentation. Cholesterol and sitosterol are the precursors for the compounds [8]. Indeed, we identified a precursor, sitosterol, that was marginally increased in the fermented AJN whereas cholesterol was not measurable. 


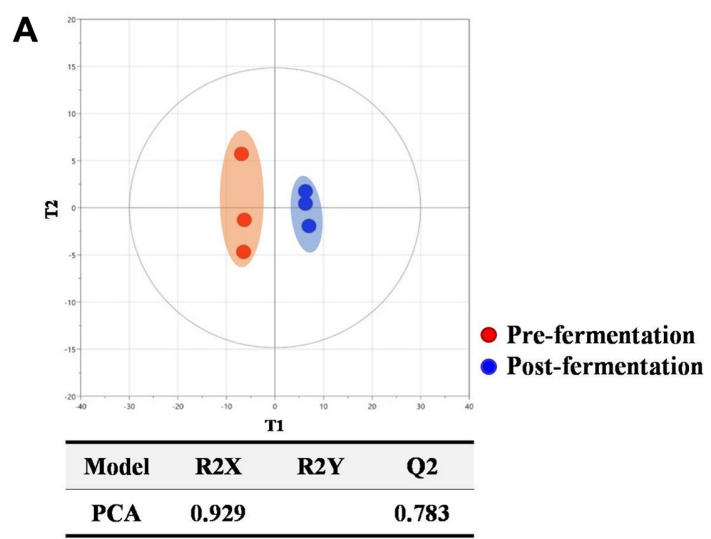

B
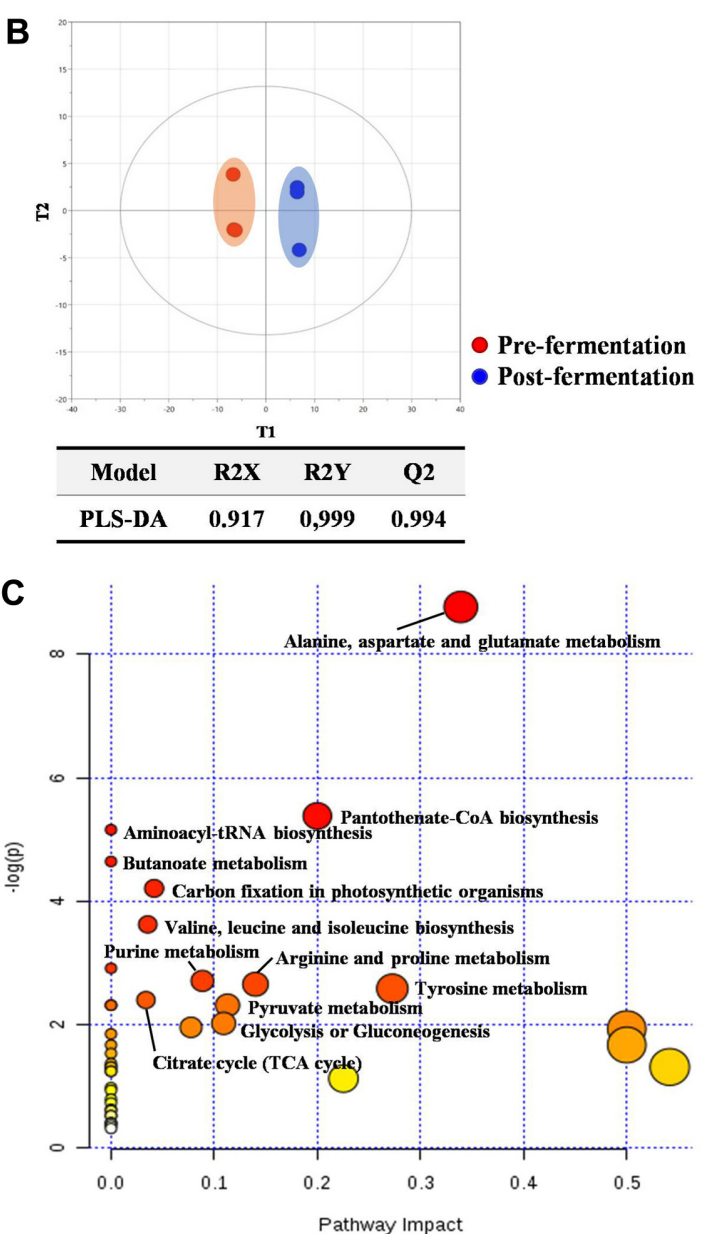

Fig. 2. Comparative analysis of primary metabolic profiles between pre- and post-fermentation.

The score scatter plots by PCA (A) and PLS-DA (B). Both unsupervised and supervised multivariate statistical analysis showed clear discrimination of the metabolite profiles between pre- and postfermentation. Pathway over-representation analysis of the metabolites that were significantly increased after the fermentation (C) $\mathrm{X}$-axis proposed the topological significance of the metabolites in a specific pathway (relative-betweenness centrality) whereas y-axis presented the significant levels of pathway alteration (hypergeometric test).
Table 1. The list of metabolites that were significantly increased by L. plantarum fermentation (Student's $t$-test, $p<0.05$ ).

\begin{tabular}{|c|c|c|}
\hline Metabolite & $p$-value & Fold change \\
\hline Cellobiose & $<0.001$ & 55.7 \\
\hline Gluconic acid & $<0.001$ & 8.2 \\
\hline Pinitol & $<0.001$ & 7.0 \\
\hline Glutamate & 0.001 & 5.0 \\
\hline Glyceric acid & $<0.001$ & 4.2 \\
\hline 3-Hydroxypyridine & $<0.001$ & 3.4 \\
\hline Isoleucine & $<0.001$ & 3.3 \\
\hline Fructose-6-phosphate & $<0.001$ & 3.3 \\
\hline Pyruvic acid & $<0.001$ & 3.2 \\
\hline Valine & $<0.001$ & 2.8 \\
\hline Fumaric acid & $<0.001$ & 2.5 \\
\hline Tyrosine & $<0.001$ & 2.4 \\
\hline 2-Deoxytetronic acid & 0.006 & 2.2 \\
\hline Tartaric acid & $<0.001$ & 2.2 \\
\hline Phenylalanine & $<0.001$ & 2.1 \\
\hline 2,3-Dihydroxypyridine & 0.011 & 1.9 \\
\hline Gamma-aminobutyric acid & 0.001 & 1.8 \\
\hline Isothreonic acid & 0.017 & 1.8 \\
\hline Xylose & 0.001 & 1.8 \\
\hline Threonic acid & $<0.001$ & 1.7 \\
\hline Hydroxylamine & 0.028 & 1.6 \\
\hline Butyrolactam & 0.002 & 1.6 \\
\hline Oxalic acid & 0.022 & 1.6 \\
\hline Uric acid & $<0.001$ & 1.6 \\
\hline Alanine & 0.007 & 1.5 \\
\hline Xanthine & $<0.001$ & 1.5 \\
\hline 3,6-Anhydro-d-galactose & 0.002 & 1.5 \\
\hline Beta alanine & 0.038 & 1.5 \\
\hline Nicotinic acid & 0.001 & 1.4 \\
\hline Thymine & $<0.001$ & 1.4 \\
\hline N-methylalanine & $<0.001$ & 1.4 \\
\hline Guanine & 0.004 & 1.4 \\
\hline Allantoic acid (dehydrated) & 0.001 & 1.3 \\
\hline Oxoproline & 0.006 & 1.3 \\
\hline Palmitic acid & 0.045 & 1.3 \\
\hline Lactic acid & 0.034 & 1.1 \\
\hline Uracil & 0.004 & 0.9 \\
\hline Succinic acid & 0.001 & 0.8 \\
\hline Galactinol & $<0.001$ & 0.7 \\
\hline Inositol-4-monophosphate & 0.047 & 0.6 \\
\hline 6-Chlorohexanol & 0.025 & 0.6 \\
\hline Tryptophan & 0.003 & 0.6 \\
\hline Glutamine & $<0.001$ & 0.3 \\
\hline Malic acid & $<0.001$ & 0.2 \\
\hline Erythritol & $<0.001$ & 0.2 \\
\hline Lyxitol & $<0.001$ & 0.1 \\
\hline
\end{tabular}

Fold change was the ratio of post-fermentation over pre-fermentation. 
In conclusion, this study demonstrated that the primary bioactive components, 20-hydroxyecdysone and 25Sinokosterone in $\mathrm{AJN}$, were significantly increased by Lactobacillus plantarum fermentation. Moreover, various types of primary metabolites (e.g. amino acids) and phytosterols (e.g. sitosterol) were identified and may require further comprehensive investigation on mechanistic understanding, which may lead to the development of a microbial production system for the highly-bioactive compound.

\section{Acknowledgments}

This work was supported by the New Faculty Startup Fund from Seoul National University (500-20190241) and the Korea Institute of Planning and Evaluation for Technology in Food, Agriculture, Forestry and Fisheries (IPET) through the High Value-added Food Technology Development Program, funded by the Ministry of Agriculture, Food and Rural Affairs (MAFRA) (grant number: 117054-3). The study was also supported by a grant from the World Institute of Kimchi funded by the Ministry of Science and ICT (grant number: KE1902-1).

Achyranthes japonica Nakai extract was provided by SK Bioland Co., Ltd.

\section{Conflict of Interest}

The authors have no financial conflicts of interest to declare.

\section{References}

1. Zhao BT, Jeong SY, Moon DC, Son KH, Son JK, Woo MH. 2012. High performance liquid chromatography used for quality control of Achyranthis Radix. Arch. Pharm. Res. 35: 1449-1455.

2. Filannino P, Di Cagno R, Gobbetti M. 2018. Metabolic and functional paths of lactic acid bacteria in plant foods: get out of the labyrinth. Curr. Opin. Biotechnol. 49: 64-72.

3. Zhao D, Shah NP. 2016. Lactic acid bacterial fermentation modified phenolic composition in tea extracts and enhanced their antioxidant activity and cellular uptake of phenolic compounds following in vitro digestion. J. Funct. Foods 20: 182-194.

4. Jung S, Lee JH, Lee YC, Moon HI. 2012. Inhibitory effects of three oleanolic acid glycosides from Achyranthes japonica on the complement classical pathway. Immunopharmacol. Immunotoxicol. 34: 213-215.

5. Lee SG, Lee EJ, Park WD, Kim JB, Kim EO, Choi SW. 2012. Anti-inflammatory and anti-osteoarthritis effects of fermented Achyranthes japonica Nakai. J. Ethnopharmacol. 142: 634-641.

6. Tao Y, Huang S, Yan J, Cai B. 2019. Determination of major components from Radix Achyranthes bidentate using ultra high performance liquid chromatography with triple quadrupole tandem mass spectrometry and an evaluation of their anti-osteoporosis effect in vitro. J. Sep. Sci. 42: 2214-2221.

7. Lee DY, Fiehn O. 2008. High quality metabolomic data for Chlamydomonas reinhardtii. Plant Methods 4: 7.

8. Nakagawa Y, Sonobe H. 2016. 20-Hydroxyecdysone. Handbook of Hormones, Comparative Endocrinology for Basic and Clin. Res. e98A-2: 560-563. 